\title{
Medizinethik und ärztliche Interessenkonflikte
}

\author{
Medical Ethics and Conflicts of Interest in Medicine
}

Autor

Institut

\section{E. Orfanos}

Professor emeritus; ehem. Direktor der Universitäts-Hautklinik und Poliklinik der Freien Universität Berlin, Campus Benjamin Franklin, Charité-Hochschulmedizin Berlin

\section{Bibliografie}

DOI $10.1055 / \mathrm{s}-0028-1119589$

Akt Dermatol 2009; 35:

157-161 @ Georg Thieme

Verlag KG Stuttgart · New York ISSN 0340-2541

Korrespondenzadresse

Prof. emer. Dr. med.

C. E. Orfanos

Sybelstraße 37

10629 Berlin

constantin.orfanos@charite.de

\section{Zusammenfassung \\ $\nabla$}

Die Ärzteschaft, einschl. der Dermatologen, wird heute durch die Möglichkeiten der medizinischen Hochtechnologie und die Ökonomisierung des medizinischen Berufs herausgefordert; sie ist vielfach überfordert. Die Krankenhäuser wandeln sich in Industriebetriebe um, die mit Profit arbeiten sollen, und der Arzt findet sich manchmal zwischen Budget und seinen ethischen Prinzipien gefangen. Der praktizierende Arzt wird zum Dienstleister, der anstatt seine Empathie und sein Wissen an Patienten, Gesundheitsleistungen an Kunden anbietet. Die Ärzte dürfen dabei nicht resignieren, sondern müssen darauf achten, dass die ärztliche Heilkunst unter dem
Diktat der Ökonomie nicht verloren geht und die Medizin ihren humanen und sozialen Auftrag erfüllt. In dieser Phase muss der Arzt selbst eine scharfe Trennung seiner primären ärztlichen von anderen, sekundären Interessen vornehmen und darauf achten, dass diese Trennung eingehalten wird. In einer offenen Debatte müsste er, mit dem Patienten an seiner Seite als Partner, sein Berufsprofil in Anbetracht der neuen Herausforderungen definieren, um sich selbst und den Arztberuf zu schützen. Medizinische Curricula sowie Sitzungen bzw. Seminare sollten die angehenden Ärzte während ihrer Aus- und Weiterbildung auf die ethischen Herausforderungen des Arztberufes gründlich vorbereiten bzw. rüsten.
Fragen des ethischen Verhaltens werden in vielen Bereichen unserer modernen, geldorientierten Gesellschaft von immer größerer Bedeutung, so auch in der modernen Medizin. Heute spricht man öfters und gern von Wirtschaftethik, Unternehmensethik, Ethik im Sport und in den Hochschulen und anderes mehr, während die Einhaltung ethischer Grundlagen im Gesundheitswesen für den klinisch tätigen Arzt zum Gegenstand täglicher Herausforderung wird. Unter anderem sind es nicht zuletzt medizinisch-ethische Interessenkonflikte, die den Arzt belasten und von ihm in ihrer Komplexität schwer zu übersehen sind [1 - 3]. Auf ein besseres Verständnis des Arztes für solche Konflikte muss hingewirkt werden, und zwar sowohl für die Kliniker als auch für die Ärzte, die in der Praxis tätig sind.

In diesem Zusammenhang mehren sich die Stimmen, die verstärkt auf die zunehmende Verflechtung der Medizin mit der pharmazeutischen Industrie hinweisen, woraus finanzielle Interessenkonflikte entstehen, und eine Unterbrechung dieser Allianz fordern. Die Ärzte werden einer ag- gressiven Werbung, z.T. unter dem Siegel der Fortbildung, ausgesetzt, die sie glauben machen will, dass alle neuen Medikamente besser sind als die alten. Bei einer Befragung in den USA haben $35 \%$ der befragten Ärzte angegeben, dass sie finanzielle Zuwendungen von der pharmazeutischen Industrie für ihre Fortbildung und $28 \% \mathrm{Ho}-$ norare für verschiedene Dienstleistungen erhielten [4-5]. Über das ärztliche Verhalten in Deutschland sind derartige Untersuchungen nicht geläufig, doch die Situation dürfte nicht viel anders sein. Bei weitem über die Hälfte der teilnehmenden Ärzte an Tagungen und Kongressen sind von der pharmazeutischen Herstellerindustrie gesponsert, und ein wachsender Teil der Redner erhält von Firmen direkt oder indirekt Honorare, sofern sie sich mit relevanten Behandlungen oder Medikamente befassen.

Mit Recht wird in Pressekreisen der sog. „medizinisch-industrielle Komplex" scharf unter die Lupe genommen und nachhaltig hinterfragt. Es mehren sich auch die Stimmen, die nicht nur die Industrie für die entstandene Situation verantwort- 
lich machen, sondern auch die Bereitschaft der Ärzte, sich in diese Abhängigkeit zu begeben, oder jedenfalls ihren fehlenden Widerstand.

Fehler des Gesundheitssystems sind eine der Ursachen für die entstandene Situation, eine andere dürfte die niedrige Schwelle bei der Ärzteschaft sein, solche Abhängigkeiten als solche zu erkennen und für unethisch zu halten. Auch die Partnerschaft der klinischen Forschung mit der Industrie muss strikter geregelt werden, und vereinzelt wurden dafür Vorschläge unterbreitet [6]. Inzwischen wurde zaghaft die provokative Frage gestellt, ob die enge Verflechtung der Ärzteschaft mit der pharmazeutischen Industrie heute so weit geht, dass die sog. Meinungsbildner praktisch die Interessen von Medikamentenherstellern vertreten [7].

\section{Konflikte und Konfliktregelung: Ethik-Kommissionen und Selbstïberwachung $\nabla$}

Als Basis des ärztlichen Handelns gilt, das primäre professionelle Ziel der Hilfeleistung und Besserung der Gesundheit des Patienten stets über alle anderen, möglicherweise konkurrierenden, Ziele bzw. Interessen Dritter zu setzen.

In der Praxis oder in der Klinik ist es unvermeidbar, dass der Arzt während seiner Tätigkeit in ständiger Berührung, zum Teil Auseinandersetzung, mit zahlreichen Akteuren steht, die andere, eigene Interessen verfolgen. Daraus entstehen häufig Konflikte. Eine permanente Interaktion, die in diesem Rahmen zu Konfliktsituationen führen kann, findet beispielsweise

a) mit den Herstellern von Medikamenten, Verbandmaterialien etc. und ihren Vertretern (Pharmaberater) statt, die ihre eigenen Produkte vertreiben und neue, meist teure einführen,

b) mit der aufwendigen technologischen Medizin, die die Budgets überaus belastet und deren ärztliche Notwendigkeit immer im Einzelfall geprüft werden muss,

c) mit den Krankenhausleitungen und Kostenträgern, die immer wieder, und teilweise zu Recht, auf Kostenreduzierungen drängen,

d) mit den kassenärztlichen Vereinigungen, die die ärztlichen Honorare von den erbrachten ärztlichen Leistungen unabhängig einschränken, und letztlich auch

e) mit den betroffenen Kranken und ihren Angehörigen, die durchaus dezidierte Wünsche und eigene Vorstellungen zum ärztlichen Prozedere haben.

Die potenziellen Konflikte und deren Tragweite, die dadurch entstehen, in vollem Umfang zu erkennen ist für den Arzt schwierig, zumal die Kontaktflächen immer größer und komplexer werden.

Parallel zu dieser Entwicklung, sicher auch als Folge davon, wurden in den Universitäsklinika, in großen Krankenhäusern und von den Ärztekammern während der letzten 2 - 3 Jahrzehnte Ethik-Kommissionen gegründet, die bei der Prüfung der ethischen Relevanz komplizierter medizinischer Zusammenhänge den Ärzten unterstützend zur Seite stehen, Ethikberatungen an Ärzte und Krankenpflegepersonal bei Bedarf anbieten und letztlich das ärztliche Verhalten im Interesse der Ärzteschaft und der Patienten überwachen sollen. Meist wird für die Urteilsfindung die kulturell überlieferte, christliche Werteskala als Basis des ethischen Arztverhaltens im Sinne einer modernen Bioethik zu Grunde gelegt, wobei für klassische medizinische Belange auch der hippokratische Kanon der Antike über Teilbereiche des ärztlichen Verhaltens bis heute als traditioneller Maßstab gilt.
Natürlich versteht jeder unter ethischem Verhalten und Bioethik etwas anderes, zumal viele Ärzte die geltende ärztliche Berufsordnung nicht oder nicht gründlich gelesen haben dürften. Der bekannte Eid des Hippokrates wurde inzwischen durch diese Berufsordnung ergänzt, worin das konfliktverdächtige Arzt-Industrie-Verhältnis in den Paragraphen $§ 32-35$ (Berufsordnung der Deutschen Ärztinnen und Ärzte, Wahrung der ärztlichen Unabhängigkeit; Stand 2004) ausdrücklich geregelt wird.

Auch die pharmazeutische Industrie hat einen Kodex für die Zusammenarbeit mit den Ärzten und Apothekern herausgebracht, mit einer Reihe wertvoller Hinweise. ${ }^{1}$

\section{Der Einzug der O̊konomie \\ $\nabla$}

Vor allem die ökonomischen Zwänge, die sich in den universitären und städtischen Krankenhäusern während der letzten Jahre als unbestrittene normative Regel durchgesetzt haben, treten als potenzielle Ursache von Konflikten auf. Die dort beschäftigten Klinikärzte sind unversehens in ein Spannungsfeld geraten [8], das ihre professionellen Interessen überschattet. Leider hat die Öffentlichkeit bisher nicht genügend Notiz davon genommen.

Basisfallwerte, Einheitspreise für Diagnose und Behandlung, "case mix“, Bettenreduzierung mit daraus folgender drastischer Reduzierung der Aufenthaltsdauer, Wiederverwendung medizinischer Einmalprodukte u.v.a. sind ethisch zu hinterfragen, zumal sie den Wunsch von Ärzten und Patienten nach einer individuellen medizinischen Versorgung konterkarieren. Zweifellos ist auch im Gesundheitswesen der Druck Kosten zu senken und Ausgaben zu balancieren legitim, doch der Wunsch nach Wirtschaftlichkeit darf nicht als Diktat wahrgenommen werden und zur Profitorientierung führen, wie es in einem Wirtschaftsbetrieb üblich ist.

Eine trennende Linie hier zu finden ist schwierig, doch die dominierende Ökonomie darf mit dem Argument der Effizienz nicht die Grundforderungen des ärztlichen Berufs aus dem Blickwinkel verdrängen. Das Wohl des einzelnen Kranken, ärztliche Fürsorge und Zuwendung, in jedem Einzelfall mit ethischem Verhalten untermauert, müssen im Vordergrund stehen.

Es kommt dazu, dass Maßnahmen, die mit dem Argument besserer Wirtschaftlichkeit im Gesundheitswesen eingeführt wurden, nicht immer zum angestrebten Ziel führten, oft weil sie in der Administration zeitraubend und personalintensiv waren, und ihrerseits Mehrkosten verursachten.

Auch die sog. aut-idem-Substitutionsregel, die für die Verschreibung von Pharmaka eingeführt wurde, hat das Problem der teuren Medikamente alles andere als gelöst. Untersuchungen haben ergeben, dass Rabattverträge für Generika abgeschlossen wurden, aber insgesamt keine nennenswerten Einsparungen erzielt werden konnten [9]. Dazu kommt, dass der Patient dabei verunsichert und sein Gesprächsbedarf mit Apotheker und Arzt größer wird. Der Arzt muss nicht selten sein Gewissen strapazieren, um die Substitutionsregel und die ausgehändigten Vergleichspräparate zu akzeptieren. Alle Beteiligten wissen, dass nicht immer offen deklarierte Rabattverträge die Wahl der herausgegebenen Medikamente beeinflussen, womit bei vielen Ärzten und Patien-

\footnotetext{
${ }^{1}$ FSA-Kodex für die Zusammenarbeit der pharmazeutischen Industrie mit Ärzten, Apothekern und anderen Angehörigen medizinischer Fachkreise vom 16.2.2004, zuletzt in der Fassung vom 18.1.2008, Bundesanzeiger vom 7.5.2008, BAnz. Nr. 68, S. 1636; FS Arzneimittelindustrie e.V., August 2008.
} 
ten Zweifel aufkommen, ob dieses Vorgehen ethisch vertretbar ist.

Ökonomische Überlegungen und industrielle Einflüsse fließen auch in den sog. ärztlichen Leitlinien zur Therapie ein. Diese sollen auf der Basis einer evidenz-basierten Medizin Kosten sparen. Wie ein Lauffeuer haben sich derartige, mehr oder weniger verbindliche Regularien, die eine Einschränkung der ärztlichen Entscheidungsfreiheit bedeuten, in vielen medizinischen Fachrichtungen etabliert, so auch in der Dermatologie. Zur Rechtfertigung wird wiederholt die Notwendigkeit einer Reglementierung zwecks „Sicherung der Qualität“ angeführt, verbunden mit der persönlichen Befürchtung, dass bei Nichtaufstellung der Leitlinie andere, weniger kompetente Individuen oder Instanzen einem zuvorkommen würden.

Was ist aber klinische Evidenz?

Im Hinblick auf die Wahl der in vielen Leitlinien empfohlenen Therapien stellen die von der Herstellerindustrie gesponserten klinischen Prüfungen die wichtigste Grundlage für die gebotene „Evidenz“ dar. Meist handelt es sich hier um statistische Überlegenheitsvergleiche neuer Behandlungen gegenüber Placebo, oder sog. Goldstandards in einer großen Gruppe von Kranken. Neuerdings werden auch „Nichtunterlegenheitstudien“ herangezogen, d. h. Prüfungen, ob ein neues Präparat mit einem anderen, etablierten gleichwertig ist, die ethisch zu hinterfragen sind $[10,11]$. In beiden Fällen wird zugunsten der einen oder anderen Behandlungsmodalität in einer Gruppe von Kranken das Ergebnis, also die Evidenz, in Prozentsätzen angegeben. Die Individualität des Kranken und die persönliche Erfahrung des Arztes, die die Basis für eine individuelle Betreuung von Kranken ist, bleiben auf der Strecke. So kommt es immer wieder vor, dass Wissen, das aufgrund von Studien als „Evidenz-basiert“ und über längere Zeit als klinischer Goldstandard gilt, von späteren Studien als falsch erkannt wird. Veröffentlichtes „Wissen“ ist gefragt, doch nach vorsichtigen Schätzungen sind nur $1 / 3$ aller Veröffentlichungen valide.

Es kommt dazu, dass die Protokolle derartiger Studien von den Marketing-Abteilungen der Hersteller in Verbindung mit Meinungsbildnern, die der Firma nahe stehen, konzipiert, geplant, an die Kliniken der Meinungsbildner und andere größere Zentren vergeben und auf Honorarbasis durchgeführt werden. Ihr Aufbau und die Prüfbedingungen entsprechen weitgehend den Vorstellungen der Hersteller, wobei eine finanzielle Verflechtung mit dem klinischen Vorgehen der Prüfärzte bzw. der Prüfzentren unvermeidbar ist, und von den Auftraggebern einkalkuliert wird.

Diese Umstände geben anderen, individuell angepassten Methoden einer sog. „alternativen Medizin“ Vorschub, die die Patienten als angenehmer und angemessener empfinden, vielfach $\mathrm{zu}$ Recht. Die über Jahrhunderte bewährte ärztliche Erkenntnis ,jeder Fall ist anders" wird aufgegeben, im schlimmsten Fall entscheidet der „Kontroller“ über die „Kostenrelevanz“, und damit auch über die Behandlung. All diese Praktiken widersprechen dem tradierten Prinzip der ärztlichen Heilkunst, wonach der Arzt nach eigenem Wissen, Können und kritischem Denken in jedem Einzelfall seine Aufgabe wahrnimmt.

Aus dem Arzt wird ein „Gesundheitsdienstleister“, der den Konsens praktiziert.

Neuerdings wird von Klinikleitungen ihren Ärzten empfohlen, bei ihrem Vorgehen genau ausformulierte „Behandlungspfade“ einzuhalten, d. h. bei definierten Gruppen von Erkrankungen diagnostische und therapeutische Schritte zu befolgen, deren $\mathrm{Zu}$ sammensetzung und Sequenz festgelegt sind. Abweichungen von diesen Pfaden sollen nur ausnahmsweise für den Arzt er- laubt sein. Derartige Behandlungspfade legen das ärztliche Vorgehen sowohl im diagnostischen Procedere als auch im therapeutischen Bereich fest, um eine exakte Kalkulation der Kosten zu ermöglichen und ihre weitere Senkung anzustreben.

Kann aber ein älterer, multimorbider Patient auf einem solchen Wege in seiner Hilfebedürftigkeit erfasst, diagnostiziert, behandelt und betreut werden? Hier scheint jegliches Verständnis einer individualisierten Medizin zu fehlen, zugunsten kostengünstiger anwendungsorientierter Listen, die allenfalls, hier mit Sicherheit zu Recht, dem Anfänger als Richtschnur hilfreich sein können. Die Befolgung derartiger Pfade, die von der Wirtschaft herrühren, kann beim komplexen Sachverhalt der medizinischen Behandlung und Betreuung ethisch vom Arzt nicht immer vertreten werden. Sie leiten den Rückfall in eine Gesundheitsmaschinerie ein, mit dem einzigen Ziel der Kostenminimierung, wobei der Patient selbst aus dem Mittelpunkt des Handelns gerät und der Arzt als Betreuer und Helfer geradezu entbehrlich wird.

Mit Recht muss man sich die Frage stellen:

Haben diese heutigen medizinischen Modelle, die der Ärzteschaft aufgezwungen werden, mit dem Erbe des Hippokrates und Asklepios noch etwas gemeinsam?

Die holistische, auf den einzelnen Kranken eingestellte hippokratische Medizin wird als Historie empfunden [12], und einer sog. „Alternativmedizin“ überlassen, die Ganzheitlichkeit und Individualität für sich beansprucht. Die alten Götter und Vorbilder erscheinen nur noch als vage Erinnerungen aus einer längst vergangenen Zeit und dienen allenfalls noch als klangvolle Namen in den Handelsregistern ( $\mathbf{A b b} \mathbf{1} \mathbf{a}$, b.)

\section{Heutiger Stand und mögliche Konsequenzen \\ $\nabla$}

Zahlreiche Aspekte der modernen Medizin dokumentieren die enge Verflechtung ökonomischer Zwänge mit der praktischen Ausübung des ärztlichen Berufes. Vieles spricht dafür, dass trotz aller Kontrollen die Interessen der Patienten noch nicht ausreichend geschützt sind; nur vereinzelt wurden Vorschläge erarbeitet, damit ärztliche Konflikte möglichst unterbleiben [2].

Natürlich ist es unstrittig, dass auch für den Arztberuf die Forderung nach Wirtschaftlichkeit gilt. Als generelle Grundlinie sollte man aber daran festhalten, dass die Ökonomie sich dem sozialen und humanen Charakter der Medizin unterordnen muss. Die Ärzte dürfen in ihrem Bemühen, dieses Prinzip durchzusetzen, nicht resignieren. Entscheidend bleibt, dass die ärztlichen Entscheidungen nach bestem ärztlichen Wissen und Gewissen, also im primären Interesse des Kranken getroffen werden, und nicht etwa im Interesse Dritter oder im eigenen Interesse [13].

Vom soziologischen Standpunkt her ist der ärztliche Beruf ein gehobener. Der Arzt baut durch sein Wissen und seine Persönlichkeit, d.h. durch die Glaubens- und Vertrauenswürdigkeit seiner Person die Vertrauensbasis zum Kranken auf und stellt auf dieser Basis ein intaktes, wirksames Arzt-Patient-Verhältnis her. Seine überzeugende Kompetenz und Verlässlichkeit sind dafür ausschlaggebend. Nur auf diesem Wege wird die ärztliche Tätigkeit erfolgreich ablaufen können, womit auch der gesellschaftliche Status des Arztes als Gelehrter und Anbieter gehobener professioneller Leistungen gewährleistet wird.

Man muss davon ausgehen, dass bei länger anhaltender Einflussnahme zugunsten untergeordneter, sekundärer Interessen die ärztliche Entscheidung beeinflusst wird, wobei fehlgeleitete Entscheidungen vom Arzt nicht immer als solche erkennbar, und möglicherweise nicht bewusst getroffen werden. Die Beeinflus- 


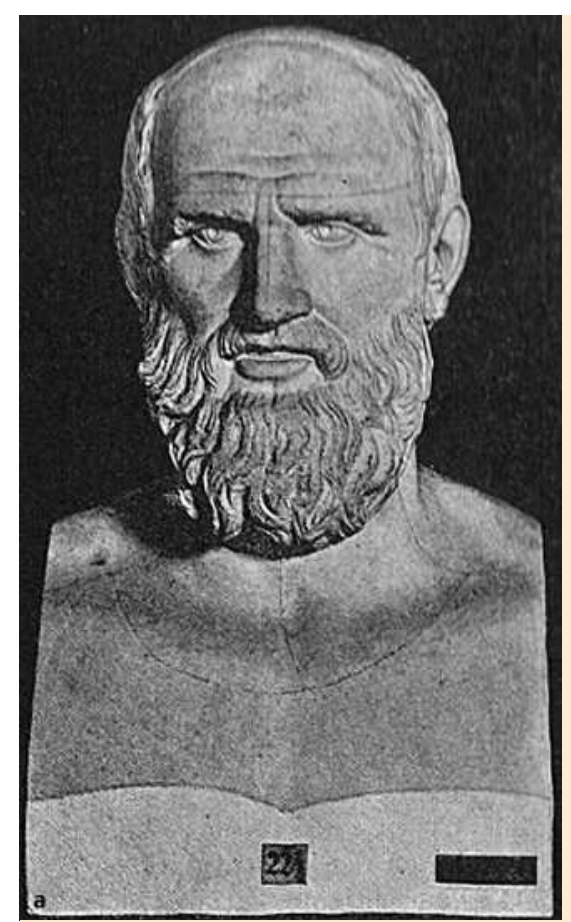

Abb. 1

a Asklepios, Sohn des Apollo, Schutzgott der Medizin und der Asklepiaden (Ärzte); Schutzfunktion erloschen? (Bild: Wikipedia)
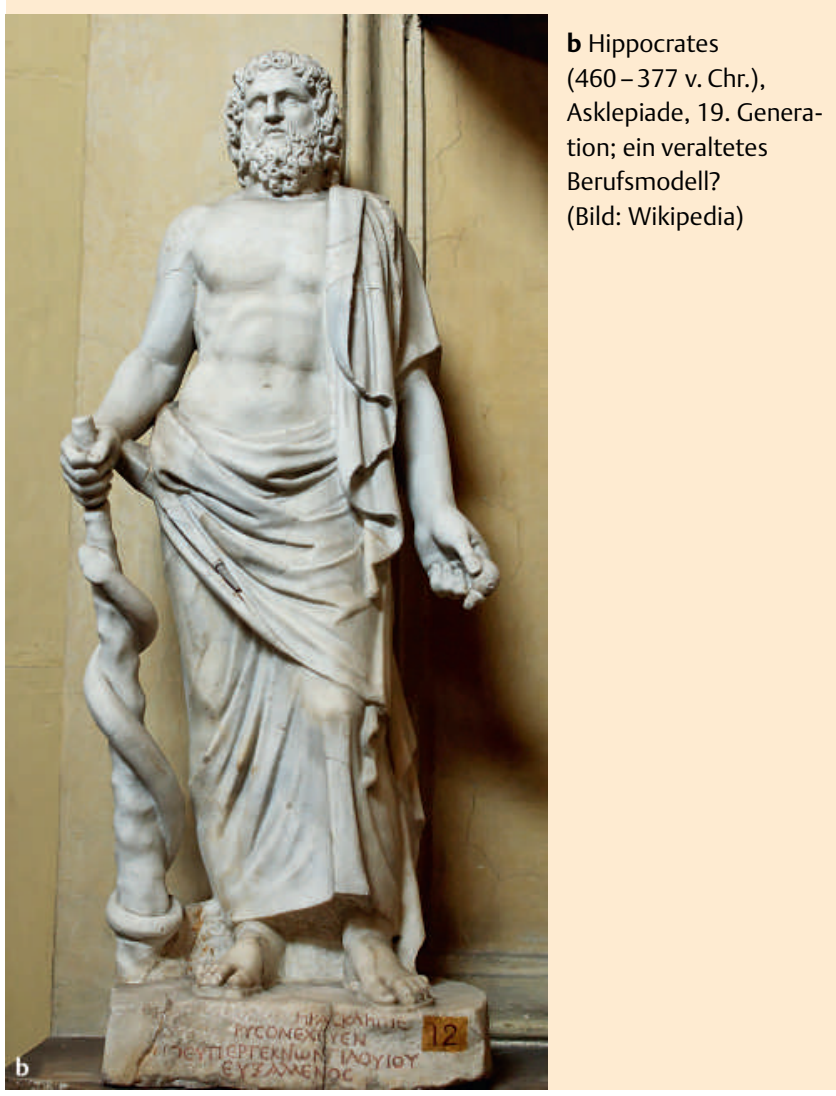

sung ist meist indirekt und wird unterbewusst wahrgenommen. Sowohl große als auch kleine Geschenke können dabei ausschlaggebend sein $[14,15]$. Als wirksames Prinzip für diese Fehlleitung soll eine Reziprozitätsregel unser Verhalten steuern [13]. Unsere jungen Ärzte müssten somit bereits während ihres Studiums und der nachfolgenden Weiterbildung auf solche sensitive Fragen und die daraus entstehenden Konfliktsituationen, mit denen sie später konfrontiert werden, vorbereitet werden. Unter den gegebenen Umständen bleiben sie ungeschützt und verhalten sie sich zumeist naiv; in der Regel negieren sie den Einfluss in ihrem persönlichen Bereich und verdrängen bewusst seine Wirkung, worauf sie zunehmend für derartige Einflüsse anfällig werden. Die Werbung dient hier als Schiene, um diese Anfälligkeit zu nutzen.

Wenn man versucht, die Gründe ärztlichen Fehlverhaltens bei Interessenkonflikten zusammenzufassen, so handelt es sich meist um Folgen,

a) eines aggressiven, zum Teil irreführenden Marketings von Seiten der Industrie (selektive Darstellung der Sachlage und der möglichen Indikation, Verharmlosung von Gegenindikationen, Nebenwirkungen, etc.), das schließlich zur aktiven Wahrnehmung positiver Werbungsinhalte führt;

b) von nachlassendem Interesse und fahrlässigem Fehlverhalten von Seiten des Arztes, verursacht durch eine nachhaltige und einseitige Werbung, die er wiederholt auf sich einwirken lässt, und schließlich

c) der Verdrängung eines korruptiven Ansatzes, bei Nichterkennung des eigenen Fehlverhaltens (,illusion of personal unvulnerability“; die anderen ja, ich nicht; [16]).

In den seltensten Fällen dürfte beim Arzt bewusstes Fehlverhalten vorliegen, d.h. um bewusste Erkennung und Akzeptanz der Korruption. Dennoch hat die Sensibilität der Ärzteschaft für moralisch-ethische Zusammenhänge offenbar kontinuierlich abgenommen, nicht zuletzt auch dadurch, dass der Arzt neuerdings zum Teil als Mediziner und zu einem anderen guten Teil als Kaufmann handeln muss (permanenter Druck zur Wirtschaftlichkeit in den Kliniken, Igel-Leistungen in der Praxis, u.v.a.). Manche fühlen sich davon überfordert, andere meinen, diese Aufgabe leicht bewältigen zu können. Die Mechanismen der selbstkritischen Konfliktüberwachung sind aber limitiert und können auf Dauer versagen [17]. Die Folge davon ist, dass die zunehmende Verflechtung von Ärzten, Ärzteverbänden und Institutionen mit Institutionen, die andere Interessen verfolgen, z. B. die Herstellerindustrie, inzwischen offen und mit nur wenig Bedenken der Öffentlichkeit vor Augen geführt wird.

Wenn aber Werbungsaktivitäten im Gesundheitswesen nicht gänzlich unterbunden werden können, müsste sich die Ärzteschaft selbst von korrumpierenden Einflüssen schützen und ihre verbliebene Resistenz stärken. Insbesondere sollte der Arzt konfliktträchtige Einflüsse meiden, um keine Situationen aufkommen zu lassen, die die ethische Basis seines Berufs gefährden. Verbindungen zu Industriefirmen, Herstellern und Vertreibern, insbesondere finanzielle Vergütungen und Vergünstigungen bei seiner Tätigkeit als fortbildungswilliger Arzt, Universitätslehrer, Vortragender und Autor müssen in vollem Umfang und im Voraus offen gelegt werden, sodass die Kranken, Studenten, Zuhörer oder Leser informiert sind und die Relevanz seiner Handlungen und Ausführungen bewerten können. Es ist strikt zu beachten, dass Verträge, die die Zusammenarbeit von Ärzten mit der Industrie regeln, nach der geltenden Berufsordnung der Ärztekammer vorgelegt werden müssen.

Ingesamt muss davon ausgegangen werden, dass Verbote in dieser komplexen Lage wenig helfen. Die Ärzteschaft muss sensibilisiert werden, mit dem Ziel, das ärztliche Verhalten ethisch stark zu untermauern. Vereinzelte Studien an der Einführung von Curricula und Seminare ethischen Inhalts scheinen Erfolg versprechend (Onkologie; [18]). Derartige ethische und Verhaltens-Seminare wären auch in der Dermatologie sinnvoll, um die Sensibilität der angehenden Ärzte auf relevante Konflikte, vor allem solche finanzieller Art, anzusprechen. Die Vorbildfunktion der Älteren wäre dabei unverzichtbar, um das aggressive Pharmamarketing einzuschränken [19]. 
Abstract

\section{Medical Ethics and Conflicts of Interest in Medicine $\nabla$}

The medical community including dermatologists is challenged today and seems overtaxed by the new roads opened through the rapid developments of medical technology and the entering of economy into the medical profession. Hospitals are transformed into entrepreneurial units, i.e. health business asked to generate profit, and the physicians are sometimes trapped between budget and their ethical principles. The physician is offering health care services to clients, instead of empathy in serving patients.

In this context the medical community should not just follow and resign, but strengthen and maintain the medical professionalism of the physician, despite the dictate of economy; the human and social mission of medicine should be maintained and carefully protected. The physicians themselves should separate their primary interest to serve the patient from other, secondary interests. The professional profile of the physician is to be defined again in an open debate, considering the new challenges, having the patient as a partner. Teaching focussed on ethics included in medical curricula and corresponding seminars would enrich and prepare the young physicians on all upcoming ethical conflicts in the medical profession.

\section{Literatur}

1 Thompson DF. Understanding financial conflicts of interest. N Engl J Med 1993; 329: 573 - 576

2 Brennan TA, Rothman DJ, Blank L et al. Health Industry Practices That Create Conflicts of Interest. A Policy Proposal for Academic Medical Centers. J Am Med Ass 2006; 295: 429-433

3 Steger F. Das Erbe des Hippokrates. Medizinethische Konflikte und ihre Wurzeln. Göttingen: Vandenhoeck \& Ruprecht, 2008

4 Campbell EG. Doctors and drug companies - Scrutinizing influential relationships. N Engl J Med 2007; 357: 1796 - 1797

5 Campbell EG, Gruen RL, Mountford J et al. A national survey of physician-industry relationships. N Engl J Med 2007; 356: 1742 - 1750

6 Stuetz A. Partnership among dermatology, the Society for Investigative Dermatology, and Industry; suggestions for change. J Invest Dermatol 2007; 127: 2487-2489

7 Moynihan R. Key opinion leaders: independent experts or drug representatives in disguise? Br Med J 2008; 336: $1402-03$

8 Görg K. Universitätskliniken - Wandel um jeden Preis? Klinikärzte im Spannungsfeld zwischen Ökonomie, Technik und Menschlichkeit. Dtsch Ärztebl 2001; 98 : A1172 - A1176

9 Meißner M. Aut-idem-Substitutionen: Mehrkosten statt Einsparungen. Dtsch Ärztebl 2008; 105 : A2144

10 Garattini S, Bartelé $V$. Non inferiority trials are unethical because they disregard patients' interests. Lancet 2007; 370: 1875- 1877

11 Meyer FP. Fragwürdige Ethik. Dtsch Ärztebl 2008; 105: A2268 - A2269

12 Orfanos C. From Hippocrates to modern medicine. J Eur Acad Dermatol Venereol 2007; 21: 852-858

13 Klemperer $D$. Interessenkonflikte: Gefahr für das ärztliche Urteilsvermögen. Dtsch Ärztebl 2008; 105: A 2098 - A2100

$14 \mathrm{Katz}$ DA, Caplan L, Merz JF. All gifts large and small. Amer J Bioethics 2003; 3: 39-46

15 Dana J, Loewenstein G. A social science perspective on gifts to physicians from industry. J Am Med Ass 2003; 290: 252 - 255

16 Sagarin BJ, Cialdini RB, Rice WE, Serna SB. Dispelling the illusion of invulnerability. The motivations and mechanisms of resistance to persuasion. J Person Soc Psychol 2002; 83: 526 - 51

17 Inzlicht $M$, Gutsell JN. Running on empty: neural signals for self-control failure. Psychol Sci 2007; 18: 933-937

18 Mehta P, Hester M, Safar AM, Thompson R. Ethics-in-oncology forums. J Cancer Educ 2007; 22: 159- 164

19 Koch K. Pharmamarketing. Millionen für Meinungsbildner. Dtsch Ärztebl 2001; 98: A 2484 